\title{
Ethical issues in randomised prevention trials
}

\author{
Nicholas Wald
}

Randomised controlled trials have made important advances in preventive medicine. It is unfortunate, therefore, that launching such trials can be a lengthy affair because of protracted discussions on ethical issues that are often misunderstood.

\section{Good ethics and good science}

One of the sources of misunderstanding arises from the failure to recognise that the key ethical question in a randomised prevention trial is also the key scientific question-namely, "Is $\mathrm{X}$ an effective and safe way of preventing disease $Y$ ?" If the answer to the question is known, there is no scientific purpose in conducting the trial, and it is also unethical to do so. If the answer to the question is not known, it is both scientifically worthwhile and ethically correct to investigate the issue, provided that the question is not a trivial one.

In research in preventive medicine, as in research in therapeutic medicine, the ethics are driven by the science; good science is a prerequisite for good ethics. The answer to the question "Is $\mathrm{X}$ an effective and safe way of preventing disease $Y$ ?" depends on making a scientific judgment based on all the relevant information available. The quality of that judgment is crucial to the ethical position. Usually, the claim that a randomised trial (whether of a preventive or a therapeutic regimen) is unethical presumes that the answer to the question that the trial is designed to answer is already known. If it is known, the claim is valid. This is not the case if there is uncertainty. It may not be an

\section{Department of Preventive Medicine, Wolfson Institute of St Bartholomew's Hospital Medical College, London EC1M 6BO \\ Nicholas Wald, professor}

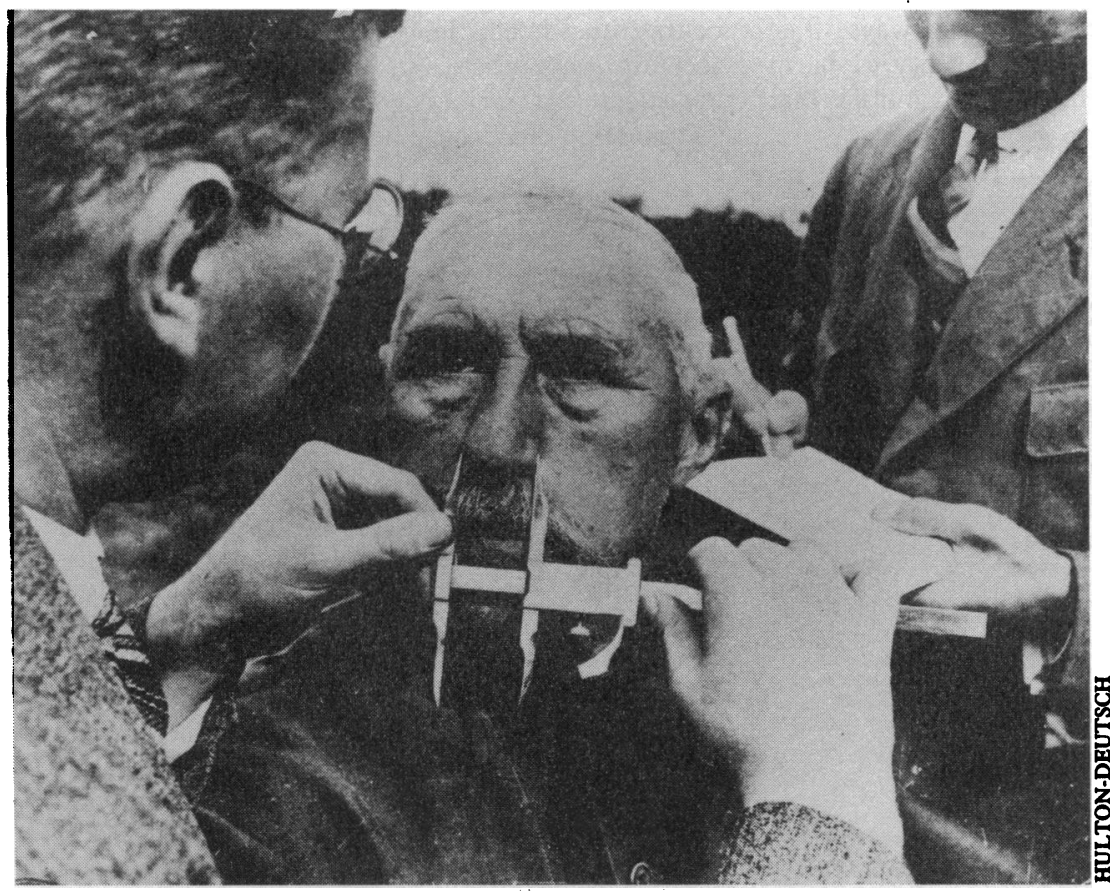

In non-preventive or non-therapeutic research the experimental subject is simply assisting the researcher to obtain biological data easy judgment to make but it is a scientific one that rests not only with researchers but with all those responsible for providing preventive and therapeutic services. The ethical debate is in fact a scientific debate. It is impossible to divorce the two. To represent an intervention as effective when its efficacy is uncertain is itself unethical.

There are difficulties in answering a question by saying that we don't know. The answer fails to distinguish between being, on the one hand, uninformed and, on the other hand, aware of enough knowledge to conclude that the question cannot be answered. The problem is exacerbated in medical practice, where the pressure to do something may be compelling and judgment may be impaired by a well intentioned but misguided presumption of benefit. For example, it has seemed self evident that if less advanced disease has a better prognosis, the earlier the disease is detected, the better the outcome. That this is not necessarily true (or if so, only to a minor extent) is illustrated by screening for lung cancer. ${ }^{1}$ The presumption of benefit bedevils much of the ethical thinking affecting proposed randomised trials.

The misunderstanding that good medicine and good science are in conflict (or worse, that there is a clash between compassion and science) may arise from a failure to distinguish medical trials from human biological research that is neither preventive nor therapeutic. In a trial each subject stands to benefit (though in the event no benefit may arise or there may even be risk of harm), but in non-preventive or nontherapeutic research the experimental subject is simply assisting the researcher to obtain biological data-for example, how a particular drug is excreted. There is a possibility, albeit unlikely, that knowledge may be advanced at the expense of the subject. Unlike in trial based research, the subject could be harmed but cannot benefit and therefore, unlike in trial based research, there is potential conflict between advancing knowledge and ensuring the safety of the individual.

\section{Evidence of efficacy}

A related misunderstanding stems from the fallacy that it is the aim of medical researchers to obtain absolute evidence of efficacy. It is perceived that researchers wish to dot the i's and cross the t's. In fact, the aim is to obtain reasonable evidence; if reasonable evidence already exists, further research is unnecessary. The legitimate debate hinges on what constitutes reasonable evidence. In formulating a view, it is helpful to examine this in two ways. Firstly, if more than one explanation could reasonably account for an apparent effect then sufficient doubt remains. Secondly, if knowledgeable doctors in good faith adopt differing practices, there is a pragmatic basis for regarding the issue as unresolved. A trial is then simply marshalling the diversity of practice in a disciplined manner capable of resolving the issue when otherwise it would remain open. The great advantage of such 
trials is that they resolve uncertainty so that an effective measure is more likely to be adopted and an ineffective one more likely to be abandoned.

Another difficulty, and indeed one implied by the original question "Is $\mathrm{X}$ an effective and safe way of preventing disease $Y$ ?", is the practice of judging the efficacy and safety of treatments as either positive or negative, rather than in quantitative terms. Estimating the extent of any benefit is as important as determining whether there is a benefit. One needs to know by how much, for example, screening for breast cancer reduces mortality from the disease in order to determine whether a screening programme is worthwhile. In terms of hazard, a procedure causing a serious complication in one out of 1000 individuals may be acceptable in clinical practice, but if in screening a procedure with the same complication rate reduced the incidence of the disorder sought by $50 \%$ in a population with an incidence of that disorder of two per 1000 , then there would be no net benefit. If the same procedure were used in a "high risk" group with an incidence of, say, 100 per 1000 then 50 would benefit for each person harmed. It should be the objective of a randomised trial to estimate the magnitude of an effect, not simply its presence.

\section{Harm}

Then there is the question of harm. Few, if any, effective interventions come without any risk of harm -but in preventive medicine the risk may be very small, whether it is a small risk of a serious adverse effect or a larger risk of a mild one. Perversely, this "low risk" situation poses its own problems. For example, the view that taking extra vitamins may be harmful does not ring true; the possibility of harm is acknowleged but it is one that is regarded as remote and pedantic. The problem is well illustrated by the debate that preceded the Medical Research Council's study on the prevention of neural tube defects. ${ }^{23}$ Even allowing for doubt about whether the vitamin supplementation was effective, administering it seems to be a one way bet - at worst it is harmless, but at best it may cure or prevent the disorder in question. This view assumes that an intervention can be biologically potent and yet non-toxic. If a chemical can affect the differentiation and growth of the developing neural tube, it could, in principle, also affect the differentiation and growth of tissues in less desirable ways. The difficulty in acknowledging the possibility of harm from a method of prophylaxis that seems natural is that

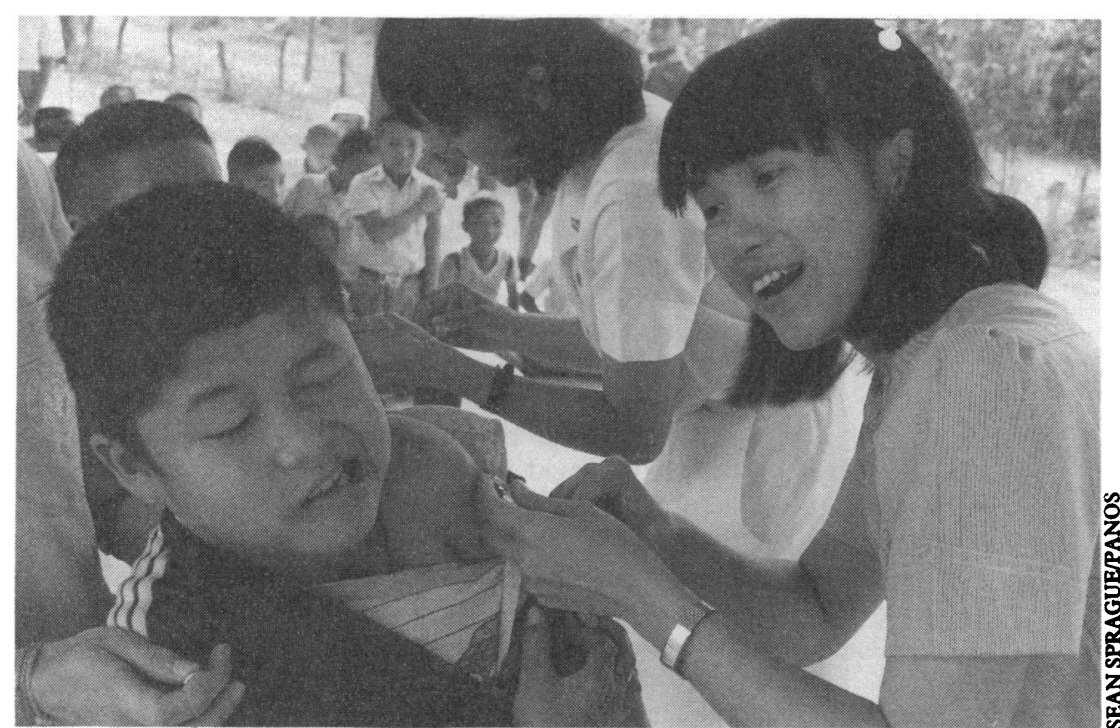

An intervention with a low probability of harm given to millions can do more damage than one with a high probability of harm given to a few the potential hazard is unspecified and hypothetical whereas the potential benefit is specific. Even if vitamin supplementation did not prevent neural tube defects, what specific harm would result? The answer that we don't know seems unconvincing.

Pointing out that there have been blunders in the past through the introduction of inadequately evaluated methods of prophylaxis (for example, oxygen for retinopathy of prematurity ${ }^{4}$ ) which, with the benefit of hindsight, would not have occurred had they been investigated properly, has all the conviction of a sermon in a secular world. It is important that our colleagues and the public recognise that although, in general, preventive strategies by their nature are likely to involve minimal risk, this does not mean zero risk; an intervention with a low probability of harm given to millions can do more damage than one with a high probability of harm given to few.

Although one of the reasons for conducting trials is the possibility of harm, trials are principally designed to test efficacy. They are weak instruments for detecting harm for two reasons. Firstly, they often recruit subjects with a high risk of the disorder under study so as to minimise the number of subjects needed to show an effect. This will reduce the statistical power of detecting adverse effects that could be quantitatively important when used in low risk situations. Secondly, since the nature of the harm is usually unknown, larger numbers are needed than if it could be specified in advance to avoid spurious statistically significant adverse effects. A two step approach is needed. A trial is first performed to show efficacy and, within limits, to quantify the effect. If efficacy can be shown then the question of harm needs to be considered by using information from the trial itself (however few the data) together with other available data; it may be necessary to set up special surveillance methods. If efficacy cannot be shown and the proposed intervention is abandoned then the question of harm becomes irrelevant.

Ironically, in the same way that the denial of the risk of harm associated with a possible preventive measure undermines the basis for performing a randomised trial designed to investigate the efficacy of that measure, the exaggeration of the risk of harm tends to undermine public health measures designed to introduce the preventive measure if it is shown to be effective. The fortification of staple foods with folic acid to prevent neural tube defects may be unwisely delayed on this account. A hypothetical risk of harm is a good reason to investigate efficacy; it is not a good reason to block the introduction of a proved benefit.

\section{Ethics and resources}

Another problem arises from the "low risk" situation in prevention trials. The general ethical approach applied to therapeutic trials, namely that there should be an approximate balance between expected benefit and possible risk to each individual in the trial (so justifying the allocation of patients at random to a new or existing treatment) is less relevant in certain prevention trials. Though the possibility of hazard should never be ignored, however remote it may seem, the ethical position in prevention trials often stems mainly from a balance between the extent of benefit (if any), and the cost of the intervention. An example is the trial currently underway to see whether breast cancer screening by mammography is better yearly than every three years. Given that mammography every three years is effective in reducing mortality from breast cancer, it follows that performing examinations yearly will be more effective. There is an increase in risk due to irradiation of the breast, but this is likely to be small and is best estimated from a woman's age and 
dose of irradiation. The issue is to estimate the marginal benefit from the increased frequency of screening examinations against the marginal increased cost. It is not an argument over which of the two screening strategies will confer the greater medical benefit. That is a foregone conclusion.

That the ethics of prevention trials is often a resource driven argument is tacitly assumed but rarely explicitly stated. It is important that the profligate use of resources is avoided; otherwise some patients will be denied a benefit because of the misdirection of limited resources. This is not to say that economy is paramount; indeed, economy is secondary to what should be an overriding principle in public health - namely, equity. We all expect to benefit equally from clean water, clean air, access to screening facilities, and so on. Given that resources are limited, a screening policy, for example, should be designed to ensure that, whatever resources are available, screening is available to all and that a fair method is adopted to designate those at highest risk as screen positive and ensure that they are offered appropriate prophylactic intervention. That this is not always the case is apparent from our policy in cervical cancer screening, the bulk of which has occurred among young women, in whom the risk of dying of cervical cancer is substantially less than among older women, who have not been the focus of public screening policies for this disease. Also, current recommendations for screening for stroke and major coronary events by blood pressure measurements are not rational in that they take little account of the absolute risk of these diseases, specifying cut off levels for blood pressure screening that take little or no account of age. The formulation of research, as well as the practice that may follow, needs to recognise explicitly the issues of equity and cost. They are important ethical considerations.

\section{Medical practice and medical research}

Ethics affect both medical practice and research, but research usually falls under the closest scrutiny. There is an important distinction between practice and research that stems principally from intent. If an intervention is established as beneficial and worthwhile it forms part of medical practice. If we are uncertain about its value and apply it mainly to assess its worth then it is research. We have, in recent years, exaggerated this distinction and created an awkward divorce between practice and research. Much of medicine is uncertain, and the admission of that uncertainty creates the obligation to research the matter further. Practice and research are intertwined; good doctors have an obligation to inquire as well as to provide a clinical service, and codes of conduct and ethical guidelines that recommend different rules for practice and research create an undesirable dichotomy that itself has ethical implications. For example, if a particular method of prevention (or treatment) is of uncertain value, there are many obstacles to be passed before the necessary research can be conducted, from seeking funds and securing the necessary collaboration to obtaining consent from many local research ethics committees. The difficulties tend to encourage the denial of the uncertainty and the assumption that the intervention is effective. It is easier to presume the benefit and call the intervention a service activity than to remain agnostic and call it research together with the inconvenient implications.

It would be desirable if randomised trials in general, and preventive trials in particular, were an integral part of medical practice, adopted whenever a new approach needed assessment and a trial was judged to be the best way of accomplishing this objective. This should be supported by the health service as part of the programme of development that any large organisation must adopt if it is to remain up to date and use its resources cost effectively. The country would consist of a network of multicentre prevention trials funded by the NHS. Apart from the need to provide the necessary explanation about each trial, they need not be considered substantially differently from other activities of the health service.

A serious obstacle to the conduct of such research, which is often necessarily large scale, is the need to consult many ethical committees. Clearly, the ethical aspects of each preventive trial need consideration, but this should be the responsibility of a central ethical committee and not a matter to be considered separately by many (perhaps over a 100) district research ethical committees that are, in the main, concerned with local clinical research projects. The view that the ethical aspects of preventive trials can only be properly considered locally is unjustified. In general, the opposite is the case: the scale of the research and the kind of expertise required to assess the scientific, and therefore the ethical, validity are such that the research is best considered centrally.

\section{Underlying principles}

Four principles therefore underlie randomised prevention trials. Much would be achieved if these were more widely acknowledged. Firstly, the ethics derive from the interpretation of science as they do in all randomised trials; secondly, quantitative estimates of efficacy are needed, to be balanced against reasonable estimates of possible adverse effects; thirdly, in prevention trials the ethics can be largely a resource based argument with equity an overriding consideration; and, fourthly, there should not be one law for practitioners and another for researchers.

The obstacles to preventive research are found in many countries, including Britain, which has a distinguished history in epidemiology, public health, and preventive medicine. In spite of this Britain still has some of the highest rates of preventable disease in the developed world, such as for ischaemic heart disease and lung cancer. Too little is done to implement existing preventive knowledge or to develop preventive strategies known to be effective. Britain has a relatively poor record of instituting randomised prevention trials; the Scandinavians, by comparison, have been more enlightened and more active in breast cancer screening. The importance of such research is not recognised in Britain. Individuals need to be persuaded that a concerted approach and a specific commitment of resources are required. In the United Kingdom, the NHS plans to spend about $£ 36$ billion in the calendar year 1993 (about $15 \%$ of public spending, or $5 \cdot 7 \%$ of the gross domestic product); a small percentage of this should be devoted to the conduct of prevention trials and the implementation of worthwhile new preventive medical services. The scale of the venture means that a nation's health service must accept a major responsibility for the work.

This article is based on a lecture given on 19 April 1991 at a symposium held in honour of Professor Geoffrey Rose on the occasion of his retirement. I thank Professor Geoffrey Rose, Professor J Dickinson, the Reverend Professor G R Dunstan, Dr Malcolm Law, and Dr and Mrs J Haddow for their helpful comments.

1 Holland WW, Stewart S. Screening in health care. London: Nuffield Provincial Hospitals Trust, 1990:140

2 Wald N. Suspended judgment-does taking extra vitamins prevent spin bifida? Eur f Paed Surg 1991;1:41.

3 MRC Vitamin Study Research Group. Prevention of neural tube defects: results of the MRC vitamin study. Lancet 1991;338:132-7.

4 Silverman WA. Retrolental fibroplasia: a modern parable. New York: Grune and Stratton, 1980.

(Accepted 16 December 1992) 